\title{
Passive Orbit Control for Space-based Geo-engineering
}

\author{
James D. Biggs * Colin R. McInnes ${ }^{\dagger}$
}

\section{Introduction}

In this Note we consider using solar sail propulsion to stabilize a spacecraft about an artificial libration point. It has been demonstrated ${ }^{1,2}$ that the constant acceleration from a solar sail can be used to generate artificial libration points in the Earth-Sun three-body problem. This is achieved by directing the thrust due to the sail such that it adds to the centripetal and gravitational forces. These libration points have the potential for future space physics and Earth observation missions. Of particular interest is the possibility of placing solar reflectors at the $L_{1}$ artificial libration point to offset natural and human driven climate change. ${ }^{3,4}$ One engineering challenge that presents itself is that these artificial libration points are highly unstable and require active control for station-keeping. Previous work has shown that it is possible to stabilize a solar sail about artificial libration points using variations in both the pitch and yaw angles. ${ }^{2,5-7}$ However, in a practical sense solar sails are large structures and active control of the sail's attitude is a challenging engineering problem. Passive stabilization of such reflectors is to be investigated here to reduce the complexity of space-based geo-engineering schemes.

A possible alternative for stabilizing a sail about an artificial libration point is to fix the attitude of the solar sail and actuate the lightness number. The sail lightness number $\beta$ is the ratio of the solar radiation pressure acceleration to the solar gravitational acceleration. This lightness number control, called $\beta$-control offers the possibility for passive stabilization of a sail about artificial libration points. $\beta$-control has previously been used to stabilize artificial libration points using it alongside a pitch angle control, ${ }^{5}$ thus active attitude control is still required. In this note we address the issue of using only $\beta$-control to stabilize a solar sail about an artificial libration point. $\beta$-control offers the possibility for passive control designs based on natural feedback laws such as $\partial \beta=-k \partial x$ where $k$ is a constant parameter, $\partial \beta$ is the change in sail lightness number and $\partial x$ is the change in distance from the Sun. One type of passive control that would naturally induce such a feedback could materialize through a conceptual 'solar balloon'. For example, as a balloon with a

\footnotetext{
*james.biggs@strath.ac.uk, Lecturer, Advanced Space Concepts Laboratory, Department of Mechanical Engineering, University of Strathclyde, Glasgow.

†colin.mcinnes@strath.ac.uk, member AIAA, Professor, Advanced Space Concepts Laboratory, Department of Mechanical Engineering, University of Strathclyde, Glasgow.
} 
reflective surface is perturbed towards the Sun away from the artificial libration point $(\partial x<0)$, the balloon would expand due to the increase in temperature thus increasing its surface area and therefore lightness number $\beta(\partial \beta>0)$. This increase in $\beta$ would then accelerate the sail back towards the artificial libration point to ensure stability. Clearly, the converse would be true if the 'solar balloon' is perturbed away from the Sun. In addition passive $\beta$-control could be realized through the use of variable reflectance materials ${ }^{8}$ or heat sensitive actuators. We do not consider details of such mechanisms in this Note.

To assess the possibility of using $\beta$-control we use the setting of the Circular Restricted Three-body Problem (CRTBP) where the massless body is a solar sail and the primaries are the Sun and Earth respectively. Firstly, we determine the $\beta$-controllability of the sail, that is we compute the controllability matrix in the vicinity of the artificial libration point assuming only lightness number control. This illustrates that the sail is $\beta$-controllable in the ecliptic plane, but not out of the plane. Secondly, we determine the $\beta$-stability of the sail, that is we compute the eigenvalues of the linearized closed-loop system to determine the linear stability properties of a $\beta$-controlled libration point. This illustrates that the libration point can be asymptotically stabilized in the ecliptic plane but out of the plane only the weaker form of Lyapunov-stability at linear order (marginal stability) can be achieved i.e. the modes associated with the imaginary eigenvalues will have bounded amplitudes. In addition a purely passive control $\partial \beta=-k \partial x$ is shown to be able to Lyapunov-stabilize a sail about an artificial libration point.

Finally, the practical implications of these results are addressed through numerical simulation. Moreover, we show that with large injection errors in the ecliptic plane, $\beta$-control can be used to asymptotically stabilize the sail at an artificial libration point. Additionally, we show that with injection errors out of the plane, $\beta$ control can be used to Lyapunov-stabilize the solar sail about an artificial libration point in the full nonlinear model (in the sense that the sail trajectory is bound in the vicinity of the artificial libration point). In the case of the passive 'solar balloon' it is shown that Lyapunov-stability can be achieved.

\section{A. Equations of motion for the solar sail}

The classical Circular Restricted Three-Body Problem (CRTBP) is modeled conveniently using a rotating frame $^{9}$ and is used as the basis for our solar sail model in the Earth-Sun system. We consider a rotating coordinate system in which the primary masses are fixed on the $x$-axis with the origin at the centre of mass, the $z$-axis is the axis of rotation and the $y$-axis completes the triad. We choose our units to set the gravitational constant, the sum of the primary masses, the distance between the primaries, and the magnitude of the angular velocity of the rotating frame to be unity. We shall denote by $\mu=3 \times 10^{-6}$ the dimensionless mass of the smaller body $m_{2}$, the Earth, and therefore the mass of the larger body $m_{1}$, the Sun, is given by $1-\mu$. Denoting by $\boldsymbol{r}, \boldsymbol{r}_{1}$ and $\boldsymbol{r}_{2}$ the position of the sail with respect to the origin, $m_{1}$ and $m_{2}$ respectively such that $\boldsymbol{r}=[x, y, z]^{T}, \boldsymbol{r}_{1}=[x+\mu, y, z]^{T} \boldsymbol{r}_{2}=[x+\mu-1, y, z]^{T}$, the solar sail's equations 
of motion in the rotating frame are

$$
\frac{d^{2} \boldsymbol{r}}{d t^{2}}+2 \boldsymbol{\omega} \times \frac{d \boldsymbol{r}}{d t}=\boldsymbol{a}-\boldsymbol{\omega} \times(\boldsymbol{\omega} \times \boldsymbol{r})-\nabla V \equiv \boldsymbol{F},
$$

with $\boldsymbol{\omega}=\widehat{\boldsymbol{z}}$ and $V=-\left[(1-\mu) / r_{1}+\mu / r_{2}\right]$ where $r_{i}=\left|\boldsymbol{r}_{i}\right|$ and $\boldsymbol{a}=\left[a_{x}, a_{y}, a_{z}\right]^{T}$. These differ from the classical equations of motion in the CRTBP by the radiation pressure acceleration term. In the case of the conceptual 'solar balloon' which for simplicity of exposition is assumed to be spherical, the radiation pressure acceleration will be in the direction of the Sun-balloon line $\widehat{\boldsymbol{r}}_{1}$ and therefore:

$$
\boldsymbol{a}=\beta \frac{(1-\mu)}{r_{1}^{2}} \widehat{\boldsymbol{r}}_{1},
$$

$\beta$ values up to 0.5 are considered and we plot all the possible artificial libration points for this $\beta$ range. This is simply computed by setting $y=z=\dot{x}=\dot{y}=\dot{z}=0$ in equation (1) and plotting $\beta$ against the artificial libration point location $\boldsymbol{r}_{e}=\left[x_{e}, 0,0\right]^{T}$ as shown in Figure 1:

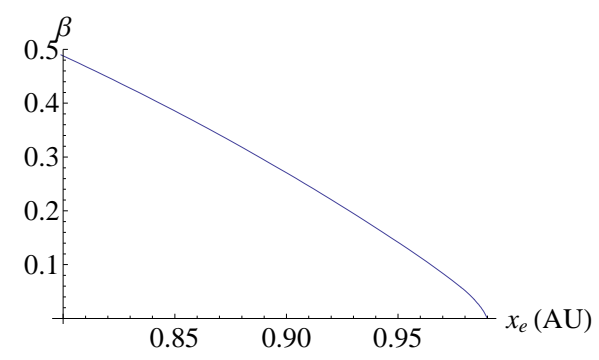

Figure 1. Plot of the sail lightness number $\beta$ against the artificial libration point in the Earth-Sun system

\section{2. $\beta$-Controllability}

In this section we determine the $\beta$-controllability of the sail about an artificial libration point. Firstly, we linearize about $\boldsymbol{r}_{e}$ by making the transformation $\boldsymbol{r} \rightarrow \boldsymbol{r}_{e}+\delta \boldsymbol{r}$, Taylor expanding $\boldsymbol{F}$ about $\boldsymbol{r}_{e}$, and neglecting the terms quadratic in $\delta \boldsymbol{r}$. Writing $\boldsymbol{X}(t)=(\delta \boldsymbol{r}, \delta \dot{\boldsymbol{r}})^{T}$ our linear system is

$$
\dot{\boldsymbol{X}}(t)=A \boldsymbol{X}(t)
$$

with

$$
A=\left(\begin{array}{c|c}
0 & I \\
\hline M & \Omega
\end{array}\right), \quad M=\left(\begin{array}{ccc}
a & 0 & 0 \\
0 & b & 0 \\
0 & 0 & c
\end{array}\right), \quad \Omega=\left(\begin{array}{ccc}
0 & 2 & 0 \\
-2 & 0 & 0 \\
0 & 0 & 0
\end{array}\right)
$$

where

$$
a=\left.\left(\partial_{x} \boldsymbol{F}^{x}\right)\right|_{e}, \quad b=\left.\left(\partial_{y} \boldsymbol{F}^{y}\right)\right|_{e}, \quad c=\left.\left(\partial_{z} \boldsymbol{F}^{z}\right)\right|_{e}
$$


For a solar sail with lightness number control we write equation (3) with a linear control term:

$$
\dot{\boldsymbol{X}}(t)=A \boldsymbol{X}(t)+B \delta \beta
$$

where

$$
B=\left[\begin{array}{llllll}
0 & 0 & 0 & \frac{\partial a_{x}}{\partial \beta} & \frac{\partial a_{y}}{\partial \beta} & \frac{\partial a_{z}}{\partial \beta}
\end{array}\right]^{T}
$$

The $\beta$-controllability of this system about the artificial libration points are then determined by the rank of the controllability matrix

$$
Q=\left[\begin{array}{llllll}
B & A B & A^{2} B & A^{3} B & A^{4} B & A^{5} B
\end{array}\right]
$$

As the rank of this $6 \times 6$ matrix is 4 , the artificial libration points are not $\beta$-controllable. However, if we set $z=0$ in equation (1) it reduces to the planar solar sail CRTBP. By linearizing the system about the artificial libration point in the planar case reveals that $Q$ is of full rank. Therefore, the libration points are $\beta$-controllable in the ecliptic plane. In the proceeding section we illustrate that despite the sail not being $\beta$-controllable out of the plane it is $\beta$-stabilizable in the sense of Lyapunov about $\mathbf{r}_{e}$.

\section{3. $\beta$-Stabilizability}

Setting $\delta \beta=0$ in (5) yields the usual CRTBP linearized about the libration point $L_{1}$. In this case the qualitative behavior in the vicinity of $L_{1}$ is representative of a saddle $\times$ centre $\times$ centre and is therefore unstable. However, in the following we assess the $\beta$-stability of the sail about the artificial libration points $\mathbf{r}_{e}$ by applying a linear feedback back control $\delta \beta=-K(\mathbf{X}(t))$ to equation (5) to yield the closed-loop system:

$$
\dot{\mathbf{X}}(t)=(A-B K) \mathbf{X}(t)
$$

If the real part of the eigenvalues of the matrix $(A-B K)$ are all less than or equal to zero then the libration points are Lyapunov-stable in the sense that the modes associated with the imaginary eigenvalues will have bounded amplitudes at linear order and if their real parts are all less than zero then they are asymptotically stable.

We initially take a near term value of $\beta=0.05$ and its corresponding artificial libration point $\mathbf{r}_{e}=$ $[0.9804099,0,0]^{T}$. Following this we define a simple gains matrix:

$$
K=-\left[\begin{array}{llllll}
\rho_{1} & 0 & 0 & \rho_{2} & 0 & 0
\end{array}\right]
$$

so that

$$
\partial \beta=-\rho_{1} \partial x-\rho_{2} \partial \dot{x}
$$

and consider two cases (i) $\rho=\rho_{1}=\rho_{2}$ and (ii) $\rho_{1} \neq 0, \rho_{2}=0$. The case where $\rho_{2}=0$ is for a purely passive control that reflects the feedback mechanism of a 'solar balloon'. 
Initially we set $\rho_{1}=\rho_{2}=\rho$ and plot the corresponding real part of the eigenvalues of the matrix $(A-B K)$ for $\rho$ up to 10 (see Figure 2). We note that the imaginary parts of the eigenvalues remain qualitatively unchanged throughout and that the real parts of $\gamma_{5}$ and $\gamma_{6}$ remain zero for all time:
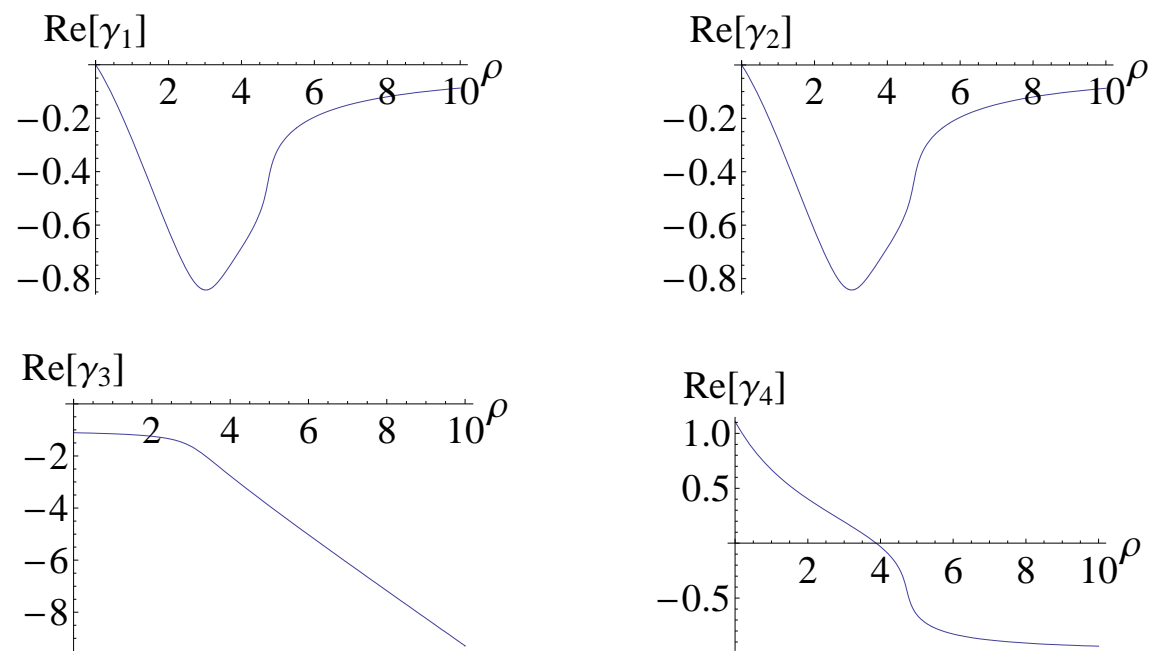

Figure 2. Real parts of the eigenvalues with increasing control magnitude $\rho$

For $\rho=0$ we have a centre $\times$ saddle $\times$ centre as in the case of $L_{1}$ with no sail acceleration. There is an instantaneous bifurcation as $\rho$ is increased above 0 ; for $0<\rho<4$ we have a stable spiral $\times$ saddle $\times$ centre. An additional bifurcation occurs at approximately $\rho=4$ and the qualitative local behavior about $\mathbf{r}_{e}$ for $\rho>4$ is a stable spiral $\times$ stable node $\times$ centre. Therefore, for values of $\rho>4$ the artificial libration point $\mathbf{r}_{e}$ is $\beta$-stabilizable in the sense of Lyapunov.

Secondly, we set $\rho_{2}=0$ and vary $\rho_{1}$ up to 10 . In this case the real parts of $\gamma_{1}, \gamma_{2}, \gamma_{5}, \gamma_{6}$ are all zero. The real part of $\gamma_{3}$ is negative for $\rho_{1}<4$ and zero for $\rho_{1}>4$ and the real part of $\gamma_{4}$ is positive for $\rho_{1}<4$ and zero for $\rho_{1}>4$. Therefore, for $\rho_{1}<4$ it is not possible to $\beta$-stabilize the artificial libration point. However, it is possible to Lyapunov-stabilize the artificial libration point for $\rho_{1}>4$ where the closed-loop local behavior in the vicinity of the equilibrium point is a centre $\times$ centre $\times$ centre. This suggests that a passive controller such as 'solar balloon' can Lyapunov-stabilize itself about an artificial libration point at linear order.

We also consider incremental values of $\beta$ up to 0.5 for both cases $\rho=\rho_{1}=\rho_{2}$ and $\rho_{1} \neq 0, \rho_{2}=0$. For each case we find that the artificial libration point $\mathbf{r}_{e}$ is $\beta$-stabilizable in the sense of Lyapunov.

To determine the linear stability of a closed-loop system of the form (6) it is also possible to use the Routh-Hurwitz stability criterion, ${ }^{10}$ however, as some or all of the eigenvalues lie on the imaginary axis this is not sufficient to prove nonlinear stability (see the Hartman-Grobman Theorem ${ }^{11}$ ). Therefore, we proceed to illustrate the nonlinear stability numerically by example. In the following section we use simulation of a near term sail $(\beta=0.05)$ at $\mathbf{r}_{e}$ to illustrate the nonlinear stability in the presence of injection errors. 


\section{Practical implications of the results}

If we place a solar sail with a near term lightness number of $\beta=0.05$ at its corresponding artificial libration point $\mathbf{r}_{e}=[0.9804099,0,0]^{T}$ it will diverge rapidly due to its inherent instability. However, using the gains matrix (7) with either (i) $\rho_{1}=\rho_{2}=10$ and (ii) $\rho_{1}=10$ and $\rho_{2}=0$, small variations in $\beta$ will maintain the sail in this position. In the following we illustrate that with large injection errors of approximately $20,000 \mathrm{~km}$ in the ecliptic plane and velocity errors in the $x-y$ directions of $200 \mathrm{~ms}^{-1}$ it is possible to asymptotically stabilize the sail onto the artificial libration point with $\rho_{1}=\rho_{2}=10$. Figure 3 (i) shows the sail's trajectory converging to $\mathbf{r}_{e}$ where $\delta x$ and $\delta y$ are the distance from the artificial libration point with the required variations in $\beta$ illustrated in Figure 3 (ii):

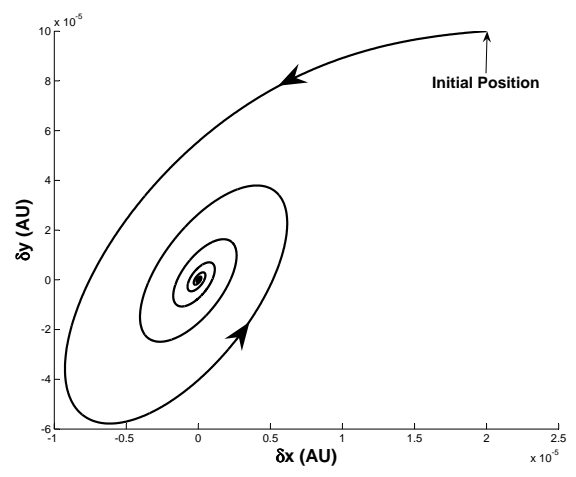

(i)

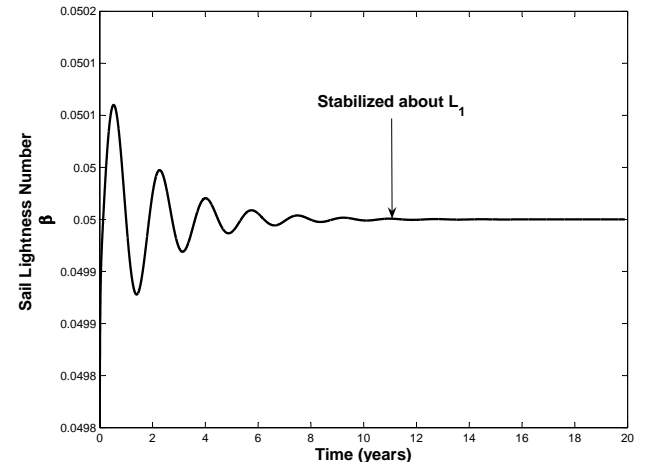

(ii)

Figure 3. (i) Sail asymptotically stabilizes about $L_{1}$ using $\beta$-control (ii) $\beta$-control variations required to drive onto and stabilize the sail about $L_{1}$

Initially as there is no injection error out of the plane the $\beta$-control can asymptotically stabilize the sail with $\rho_{1}=\rho_{2}=10$. However, the sail is not $\beta$-controllable out of the plane and implementing the same injection errors as before with a position error of $15,000 \mathrm{~km}$ in the $z$ direction yields the variations in the $x-z$ plane as illustrated in Figure 4 (i) where $\delta x$ and $\delta z$ are the distance from the artificial libration point. In the case of the purely passive control with $\rho_{1}=10, \rho_{2}=0$ asymptotic stability is not possible in any direction, but it does stabilize about the artificial Libration point in the sense of Lyapunov (the trajectory is bounded) as shown in Figure 4 (ii).

In Figure 4 (i) the $\beta$-control asymptotically stabilizes the sail in the ecliptic plane as in Figure 3 (i) but oscillates in the $z$ direction about $\mathbf{r}_{e}$ with an amplitude equal to the initial position error in the $z$ direction (defined as $\epsilon$ ). Therefore, the sail can only be $\beta$-stabilized in the sense of Lyapunov $\left(x \rightarrow x_{e}, y \rightarrow 0, z \leq \epsilon\right)$. For geo-engineering purposes the initial $z$ error must be less than the radius of the solar disk. In the case of complete passive control required for the implementation of a 'solar balloon' asymptotic stability is not 


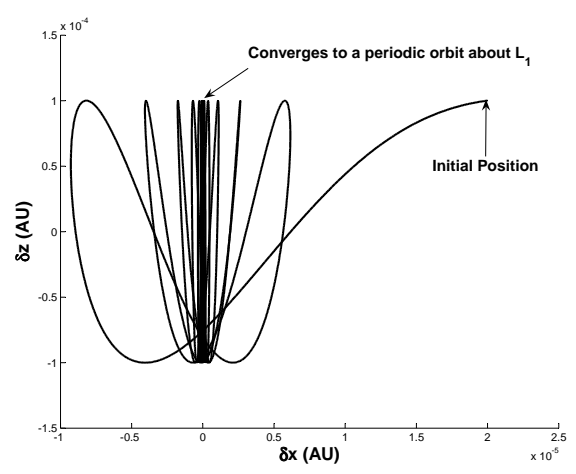

(i)

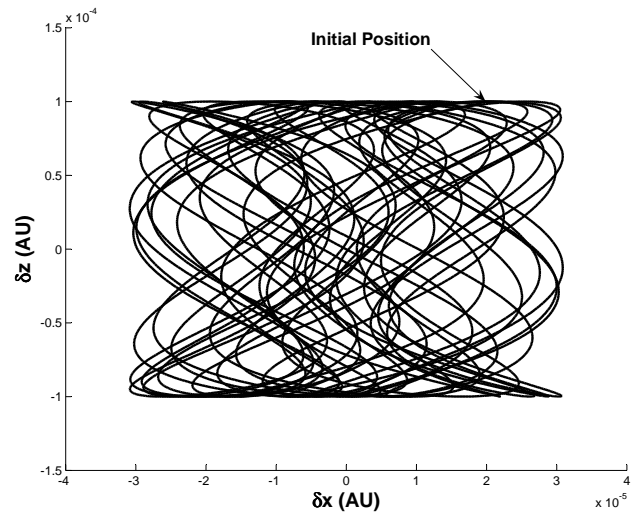

(ii)

Figure 4. (i) $\rho_{1}=\rho_{2}=\rho=10$ Sail converges to a periodic orbit about $L_{1}$ where the initial injection error in the $z$ direction is equal to the amplitude of oscillation (ii) $\rho_{1}=10, \rho_{2}=0$ the sail is Lyapunov stabilized about the libration point

possible in any direction but Lyapunov stability is. Therefore, the effectiveness of a purely passive $\beta$-control is strongly dependent on the initial injection errors.

\section{Conclusion}

This Note has illustrated that a solar sail may stabilize its motion in the sense of Lyapunov about an artificial libration point using only small variations in its lightness number $\beta$. It has also been shown that the sail is $\beta$-stabilizable in the sense of Lyapunov about the artificial equilibrium point despite the fact it is only $\beta$-controllable in the plane. The implications of this are illustrated in simulation and we show that in the presence of injection errors in the ecliptic plane it is possible to asymptotically stabilize the sail. However, if there are injection errors in the $z$ direction the results illustrate that it is only possible to Lyapunov-stabilize the sail about the artificial libration point. In addition it is illustrated that in the purely passive case, for example using a 'solar balloon' it is possible to achieve only Lyapunov-stability if any injection error occurs.

\section{Acknowledgments}

This work was funded by grant EP/D003822/1 from the UK Engineering and Physical Sciences Research Council (EPSRC) and by the European Research Council grant 227571 VISIONSPACE.

\section{References}

\footnotetext{
${ }^{1}$ McInnes, C. R., 'Solar sailing: technology, dynamics and mission applications'. Springer Praxis, pp. 17-19, 1999.

${ }^{2}$ McInnes, C.R.,McDonald, A.J.C., Simmons, J.F.C., and McDonald, E.W., 'Solar sail parking in restricted three-body
} 
systems'. Journal of Guidance, Control and Dynamics, Vol. 17, No. 2, pp. 399-406, 1994.

${ }^{3}$ McInnes, C.R. 'Minimum mass solar shield for terrestrial climate control'. JBIS, Journal of the British Interplanetary Society, 55 (9-10). pp. 307-311. 2002

${ }^{4}$ Angel, R. 'Feasibility of cooling the Earth with a cloud of small spacecraft near the inner Lagrange point $\left(L_{1}\right)$ '. PNAS, vol. 103, no. 46, pp. 17184-17189. 2006 .

${ }^{5}$ Baoyin, H., McInnes, C.R., 'Solar sail Orbits at Artificial Sun-Earth Libration Points'. Journal of Guidance, Control and Dynamics, Vol. 28, No. 6, pp. 1328-1330, 2005.

${ }^{6}$ Bookless, J., McInnes, C.R., 'Control of Lagrange point orbits using solar sail propulsion'. Acta Astronautica, Vol. 62, pp. 159-176, 2008 .

${ }^{7}$ Waters, T. J., McInnes, C. R., 'Periodic Orbits above the Ecliptic in the Solar sail Restricted Three-body problem'. Journal of Guidance, Control and Dynamics, Vol. 127, pp. 1947-1960, 2007.

${ }^{8}$ Keselbrener, M., Ruschin, S., 'Interferometric variable reflectivity mirror: experimental results' Optical Materials 13, pp. 97-100, 1999.

${ }^{9}$ Szebehely, V., 'Theory of Orbits: The restricted problem of three bodies'. Academic Press, New York, pp. 587-595, 1967.

${ }^{10}$ Gantmacher, F. R. 'Applications of the Theory of Matrices'. New York: Wiley, p. 230, 1959.

${ }^{11}$ Strogatz, S. H. 'Nonlinear Dynamics and Chaos'. Studies in Nonlinearity, Westview Press, p. 155, 2000. 